\title{
Evaluación de las características relacionadas con la absorción de agua para diversas piedras de España tratadas con productos de conservación
}

\section{Evaluation of the water related characteristics of diverse Spanish stones after the application of different protective treatments}

2. RUBYO Y M.A. BELLO

Depto. de Química Analitica, Facultad de Química, Univ. de Sevilla

\section{RESUMEN}

Las caracteristicas relacionadas con la absorción de agua de algunas de las más importantes piedras usadas en el sur de España han sido estudiadas antes y tras la aplicación de diversos tratamientos de protección comerciales, incluyendo la aplicación con diluciones diversas y la aplicación sucesiva de dos productos complementarios.

Se exponen diversas consideraciones acerca de la adecuación de los pares producto/piedra.

\section{SUMMARY}

The characteristics related to water absorption of some important stones very used in the southern of Spain were tested before and after the application of diverse commercial protective treatments, including the application of diverse dilutions and the correlative application of two complementary products.

Some considerations about the adequation of the pair product/stone have been realized.

\section{INTRODUCCIÓN}

El agua es un factor de alteración de gran importancia en los procesos de degradación de materiales pétreos (1-3); así, los procesos químicos de alteración sólo son apreciables en presencia de agua, y si el agua no está presente, las reacciones químicas entre los componentes de la piedra no son posibles, no ocurren los procesos de migración y de solubilización/cristalización de las sales solubles presentes, y la acción de los contaminantes atmosféricos es de escasa importancia. Asimismo, el agua provoca ciertos procesos de alteración que se deben exclusivamente a su propia naturaleza: disgregación por solubilización, fracturas por formación de hielo dentro de los poros, etc.

El agua puede acceder a la piedra a través de diferentes mecanismos: lluvia, percolación, ascensión capilar,

\section{INTRODUCTION}

Water is a very important alteration factor in the degradation processes of stony materials (1-3); so, the chemical processes of alteration on the stones are only possible in presence of water; if water is not present: chemical reactions between the stone components are not possible, the migration and solubilization/ crystallization processes of the water-soluble salts do not can to occur, and the action of the atmospheric pollutants are of small importance. Likewise, the water provokes alteration processes due to its nature: dissagregation by solubilization, cracking by ice formation into the pores, etc.

Water can to access to the stone through different mechanisms: rain, water percolation, capillar ascent, 
humedad ambiental, etc, que tienen, a veces, una gran influencia en el tipo y grado de alteración producido (4). En relación con estos aspectos, se han diseñado diversos ensayos de laboratorio para evaluar el comportamiento de los materiales pétreos frente al agua (5).

De acuerdo con lo expuesto hasta ahora, resulta clara la importancia de reducir el acceso del agua a la piedra con el objetivo de minimizar su acción directa y/o sinérgica en la alteración de los materiales pétreos. Por esta razón, son muy numerosos los productos de conservación comerciales que tienen características hidrófugas, única o asociada a otras tales como consolidación, oleofugación, etc. En general, todos aquellos productos con características hidrofóbicas provocan un cambio en las propiedades relacionadas con la absorción de agua en los materiales en que son aplicados.

En la bibliografía se encuentran profusamente descritos los efectos deseables en un producto de conservación y en los materiales tratados, y el más frecuente punto de interés se centra en el estudio de la variación de las características relacionadas con el agua.

En este artículo se chequean algunos de los más importantes tipos de piedras usados en monumentos del Sur de España, así como productos comerciales de conservación que se podrían considerar como representativos de los principales tipos existentes, y para ello se han evaluado los cambios producidos en las propiedades relacionadas con el agua tras la aplicación de los diversos tratamientos. Asimismo, ha sido también objeto de estudio la aplicación correlativa de dos productos de conservación de características complementarias.

\section{PARTE EXPERIMENTAL}

Para la determinación de la composición química mayoritaria de las piedras estudiadas se ha empleado la metodología propuesta por Berzosa y Martín [6-8].

Los parámetros hídricos más significativos han sido determinados de acuerdo con las recomendaciones del RILEM [5], y se emplearon para los ensayos probetas cúbicas de $5 \mathrm{~cm}$ de arista.

\subsection{Materiales Pétreos Estudiados}

Para este trabajo, se estudiaron seis materiales muy usados en monumentos andaluces.

De las canteras de Almería, situadas cerca de la ciudad, se estudiaron dos calizas dolomíticas de apariencia muy air humidity, etc which are sometimes great influence on the type and degree of deterioration produced (4). In this way, some laboratory tests for the evaluation of the behaviour of the stones to the water have been designed (5).

According to the water effects previously described, it is important to reduce the access of the water to the stone with the aim to minimize its direct and/or synergic action on the stone weathering. For this reason, there are numerous commercial products of conservation for the stone which have water repellent characteristics, only or associated to other characteristics just as consolidation, oil repellency, etc. Even those products without hydrophobic characteristics provoke a change on the characteristics related to water absorption of the treated materials.

The desirable effects of the conservation products on all the characteristics of the treated stones have been profusely described in the bibliography, and the most frequent interesting point is the study of the variation on the water-related characteristics.

In this work, some of the most important stone-types used in the monuments of the Southern of Spain and representative commercial products of conservation have been checked with the aim to evaluate the changes produced on the water related properties of the treated materials. Likewise, the correlative application of two products of conservation with complementary characteristics has also been tested.

\section{EXPERIMENTAL}

For the determination of the major composition of the stones, the Berzosa and Martin methodology [6-8] was used.

The most significant hygric parameters were determined according to RILEM proposal[5]. Cubic specimens of $5 \mathrm{~cm}$ edge have been used.

\subsection{Stony Materials Tested}

For this work six stone types very used andalusian monuments were tested.

Two dolomitic limestones from Almeria quarries, located near the city, of very similar appearance, one 
similar, una de color blanco-grisáceo y otra de tono amarillento; la porosidad abierta de estos materiales oscila entre 44,5-48,5\% para la variedad "blanca" y el $39-43 \%$ para la de color amarillento. Otro de los materiales estudiados es una arenisca calcítica de las canteras del Puerto de Santa María (Cádiz), de color blanco-amarillento y porosidad entre $33-37 \%$. Los materiales procedentes de Padul (Granada) son calizas de porosidad entre el 26 y el $31 \%$ y color amarillento. La piedra denominada Martelilla es una caliza de color amarillo y porosidad entre $20-31 \%$, cuyas canteras se encuentran cerca de Jerez de la Frontera (Cádiz). El ultimo material estudiado es una arenisca calcítica de la cantera de Baeza (Jaén), de color marrón y porosidad entre el 25 y el $28 \%$.

Los materiales de las canteras de Almería constimyen la práctica totalidad de la catedral de esa ciudad, y además se encuentran profusamente distribuidos en el resto de monumentos de la ciudad. Los materiales procedentes de las canteras del Puerto de Santa María han sido ampliamente usados en numerosos monumentos andaluces, pudiendo destacarse su uso en la catedral de Sevilla, el monumento gótico mayor del mundo. La catedral de Granada, la mayor catedral renacentista de España, está prácticamente realizada en su totalidad con materiales de Padul, material que también ha sido usado en otros monumentos de Granada. La piedra martelilla ha sido usada en algunos monumentos de Cádiz. Los materiales de la cantera de Baeza han sido ampliamente usados en las ciudades y pueblos cercanos a la cantera.

En todos los casos se trata de materiales muy porosos, lo que contribuye a la absorción de agua, por lo que es muy frecuente la existencia de procesos de alteración asociados al movimiento de agua en aquellos monumentos donde han sido empleados estos materiales $(4,9-10)$.

En la Tabla I se puede observar la composición química mayoritaria de los materiales pétreos estudiados. of these of white-greyish color and the other of yellowish color, the open porosity for these materials are $44.5-48.5 \%$ (white variety) and $39.43 \%$ (yellow variety). Other stone is a calcitic sandstone from Puerto de Santa Maria quarries (Cadiz) of whiteyellowish color and open porosity of $33.37 \%$. The materials from Padul (Granada) are limestones of porosity of 26-31\%, yellowish color. Martelilla stone is a limestone of yellow color and porosity betweer 20-31\%, which quarries are located near Jere de la Frontera (Cadiz). The last material is a calcitic sandstone from Baeza quarry (Jaén) of brown color and open porosity of $25-28 \%$

The two first materials, those from Almeria; constitute the practical totality of the Almeria Cathedral and they are profusely distributed in the rest of monuments of the city. The materials from Puerto de Santa Maria quarries have been widely used in numerous andalusian monuments, it is remarkable their use in the Sevilie cathedral, the biggest gothic monument. The Padul materials constitute the practical totality of the Granada cathedral which is the biggest renaissance cathedral of Spain, and they also widely were used in other monuments of Granada. The Martelilla stone was used in some monuments of Cádiz, and the Baeza stone was widely used in the cities and villages near the quarries.

In all the cases they are very porous materials which contributes to the water absorption and it's very frequent the existence of water-associated deterioration processes in the monuments where those materials have been used (4,9-10).

Table I shows the typical chemical major composition of the stony materials tested in this work.

TABLA I (TABLE I)

Composición química mayoritaria (\%) típica de los materiales pétreos estudiados (Typical major chemical composition (\%) of the stony materials tested)

\begin{tabular}{||l|llllllllll||}
\hline $\begin{array}{l}\text { PIEDRA } \\
\text { (STONE) }\end{array}$ & $\begin{array}{l}\text { Pérdida } \\
\text { (loss on } \\
\text { ignition) }\end{array}$ & $\mathrm{SiO}_{2}$ & $\mathrm{Al}_{2} \mathrm{O}_{3}$ & $\mathrm{Fe}_{2} \mathrm{O}_{3}$ & $\mathrm{CaO}$ & $\mathrm{MgO}$ & $\mathrm{Na}_{2} \mathrm{O}$ & $\mathrm{K}_{2} \mathrm{O}$ & $\mathrm{SO}_{3}$ & Total \\
\hline Almería white & 43.66 & 8.55 & 1.77 & 1.23 & 27.58 & 16.20 & 0.36 & 0.03 & 0.98 & 100.36 \\
Almería yellow & 43.05 & 7.69 & 1.31 & 1.80 & 32.02 & -12.35 & 0.25 & 0.03 & 1.28 & 99.78 \\
Puerto Sta. María & 27.78 & 36.19 & 0.28 & 0.17 & 35.19 & 0.22 & 0.03 & 0.01 & 0.43 & 100.30 \\
Baeza & 29.75 & 30.78 & 1.09 & 1.49 & 30.87 & 5.24 & 0.12 & 0.06 & 0.68 & 100.08 \\
Martelilla & 41.14 & 3.35 & 0.35 & 1.25 & 53.00 & 0.12 & 0.25 & 0.03 & 0.73 & 100.22 \\
Padul & 42.17 & 5.75 & 0.78 & 0.74 & 49.16 & 0.15 & 0.30 & 0.04 & 1.02 & 100.11 \\
\hline
\end{tabular}




\subsection{Productos de Conservación}

Para este estudio se han chequeado seis conocidos productos de conservación; en algunos casos también se ha estudiado el efecto de la aplicación a diversas diluciones, así como la aplicación correlativa de dos productos complementarios. Cuatro de los productos son de tipo silicónico: Stone Strengthener OH (silicato de etilo), Tegovakon V (silicato de etilo), Masonry Water Repellent BS28 (organosilícico), Tegosivin HL100 (monómero organo-silícico); un producto acrilsilicónico: ARD 55.050 (resina acrilsilicónica) y uno acrílico: Paraloid B72 (copolímero de metilacrilato y etilmetacrilato).

Respecto a su efecto sobre la piedra, pueden clasificarse en:

\footnotetext{
* Consolidantes: Strengthener $\mathrm{OH}(\mathbf{O H})$ y Tegovakon V (TV)

* Consolidantes + hidrófugos: Paraloid B72 (PA) y ARD 55.050 (ARD)

* Hidrófugo: Tegosivin HL100 (TG)

* Hidrófugo y oleófugo: BS28
}

Estos productos cubren los principales tipos en composición y efectos, y fueron aplicados sobre piedras inalteradas procedentes de la canteras respectivas.

\subsubsection{Aplicación de los Productos sobre la Piedra}

Todos los ensayos se realizaron sobre probetas cúbicas de piedra de $5 \mathrm{~cm}$ de arista. Antes de la aplicación de los productos, las muestras se limpiaron, pesaron, y se dejaron secar al aire hasta que el contenido de agua oscilara entre 0-2 gramos; este procedimiento reproduce el estado de los materiales pétreos en el edificio y favorece la polimerización de los productos organosilícicos (11-13). En la Tabla II se muestran los disolventes y diluciones usadas para la aplicación de los productos de conservación objeto de estudio.

Para cada par piedra/producto se trataron tres probetas por inmersión, durante diez minutos, al objeto de conseguir una penetración uniforme en todas las caras. Debido a la alta porosidad de las piedras ensayadas, este tiempo es suficiente para conseguir la impregnación total de las probetas. Para los casos de la aplicación correlativa de dos productos de conservación, se aplicó, en primer lugar, el producto consolidante, y una vez seco éste, se procedió a la aplicación del otro producto; se ensayaron dos parejas: Strengthener OH + BS28 (OB) y Tegovakon V + Tegosivin HL100 (10\%) (TT). Tegosivin fue aplicado también a dos diluciones distintas: $5 \%$ (TG) y 10\% (TGI).

\subsection{Products of Conservation}

For this study, six known commercial products of conservation were tested; in some cases, diverse dilutions and the application of two products were also tested. Four products are of silicone type: Stone Strengthener $\mathrm{OH}$ (ethylsilicate), Tegovakon $\mathrm{V}$ (ethylsilicate), Silicone Masonry Water Repellent BS28 (organosilicic), Tegosivin HL100 (monomeric organosilicic); one acrylsiliconic product: ARD 55.050 (acrylsiliconic resin) and one acrylic product: Paraloid $B 72$ (copolymer of methylacrylate and ethylmethacrylate).

Respect to their effect on the stone, they can be classified in:

${ }^{*}$ Consolidants: Strengthener $\mathrm{OH}(\mathrm{OH})$ and Tegovakon $\mathrm{V}$ (TV)

* Consolidants + water repellents: Paraloid B72 (PA) and ARD 55.050 (ARD)

* Water repellents: Tegosivin HL100 (TG)

*Water and oil repellent: BS28

These products cover the main types in composition and effects, and were applied on unaltered stones from the respective quarries.

\subsubsection{Application of the Products on the Stone}

Cubic samples of $5 \mathrm{~cm}$ edge were used for all the tests carried out. Before applying the products, the samples were cleaned, wetted and left in the air to dry until the water content was 0-2 $\mathrm{g}$; this procedure reproduces the state of the stone in the building and favours the polymerization of organosilicic products (11-13). Table II shows the solvents and dilutions used for the application of the conservation products.

Three samples were treated by ten minutes immersion in order to reach an uniform penetration on all faces. Due to the high stone porosity, this time is enough to achieve a total impregnation of the samples. For the cases of correlative application of two conservation products, first the consolidating product was applied and when the drying process was finished the other product was applied too; two mixes were tested: Strengthener $\mathrm{OH}+\mathrm{BS} 28(\mathrm{OB})$ and Tegovakon V+ Tegosivin HL100 (10\%) (TT). Tegosivin was also applied in two dilutions: $5 \%(T G)$ and $10 \%$ (TGI). 
Disolventes y diluciones usadios para los productos aplicados (Soivents and disolutions used for the products applied)

\begin{tabular}{|l|l|}
\hline PRODUCTO (PRODUCT) & DILUCIÓN (DILUTION) \\
\hline Strengthener OH & Listo para uso (Ready to use) \\
Tegovakon V & Listo para uso (Ready to use) \\
BS28 & $5 \%(v / v)$ with xylene \\
Tegosivin HL100 & 5 and 10\% (v/v) with xylene \\
ARD 55.050 & Listo para uso (Ready to use) \\
Paraloid B72 & $5 \%(w / v)$ with 1, 1, 1-trichlorethane \\
\hline
\end{tabular}

Al objeto de poder monitorizar el proceso de secado/polimerización, las probetas tratadas fueron pesadas periódicamente; este proceso depende de la evaporación del disolvente y de la polimerización del compuesto activo (si es un monómero u oligómero).

En la Tabla III se pueden apreciar diversas características de las probetas tratadas.

Debido al carácter monumental de los edificios, donde estos materiales pétreos han sido usados, es importante chequear los posibles cambios de coloración que pudiera producir la aplicación de los productos de conservación: En la Tabla IV se relaciona el grado de oscurecimiento observado en los materiaies pétreos tras el tratamiento.

\section{RESULTADOS Y DISCUSIÓN}

De acuerdo con la Tabla III, se pueden realizar diversas consideraciones. El tiempo de secado de los productos para las diversas piedras estudiadas es muy similar, los productos que tienen sólo carácter consolidante tienen los mayores tiempos de secado, y el menor tiempo de secado corresponde, siempre, a Paraloid B72, lo que puede deberse al hecho de que este producto se comercialice en forma de copolímero.

El incremento de peso debido al producto activo absorbido oscila, dependiendo del par piedra/producto, entre un 1 y un $11 \%$; no obstante, como cabía esperar, el mayor incremento de peso se produce tras la aplicación
The samples were periodically weighted with the aim to monitorize the drying/polymerization process, which depends of the solvent evaporation and polymerization of active compound (if it's a monomer).

Table III shows diverse characteristics of the treated samples.

Due to the monumental character of the buildings that the stony materials have been used, it is very important to check the coloration changes that the conservation products can produce. Table IV summarizes the degree of darkening observed after the treatment (if some one has been occurred).

\section{RESULTS AND DISCUSSION}

According to Table III, some considerations can be realized. The drying time of the products for the different stones was very similar, the products with character consolidant only have the higher drying times and the lower time always corresponds to Paraloid B72, which can be due that this product is manufactured as copolymer.

The weight increase due the applied product, depending to the pair stone/product, oscillates between I and $11 \%$ approximately; however, as might were expected, in general the higher increases occur when 
TABLA III (TABLE III)

\begin{tabular}{|c|c|c|c|c|c|c|c|c|}
\hline & \multicolumn{6}{|c|}{$\begin{array}{l}\text { Diversas características de las piedras tratadas } \\
\text { (Diverse characteristcs of the treated stones) }\end{array}$} & & \\
\hline & \multicolumn{8}{|c|}{ PRODUCTO APLICADO (PRODUCT APPLIED) } \\
\hline & PA & $\mathrm{OH}$ & TV & TG & TGI & ARD & OB & TT \\
\hline \multicolumn{9}{|l|}{ ALMERIA WHITE } \\
\hline Tiempo de secado (Time of drying) (h) & 120 & 840 & 672 & 312 & 336 & 192 & $648^{\circ}$ & $912^{\prime \prime}$ \\
\hline Incremento de peso (Weight increase) (\%) & $7.04 \pm 6.01$ & $6.30 \pm 2.44$ & $8.32 \pm 4.38$ & $1.50 \pm 0.22$ & $2.90 \pm 0.53$ & $1.37 \pm 0.23$ & $10.81 \pm 0.58$ & $10.72 \pm 0.66$ \\
\hline Descenso de porosidad (Porosity decrease) ( $\Delta \%$ ) & $1.51 \pm 0.14$ & $3.84 \pm 1.21$ & $5.33 \pm 2.27$ & $2.04 \pm 0.28$ & $3.71 \pm 0.96$ & $2.24 \pm 0.21$ & $6.74 \pm 1.23$ & $9.19 \pm 2.73$ \\
\hline Incremento de densidad (Density increase) $\left(\mathrm{g} \mathrm{cm}^{-3}\right)$ & $0.05 \pm 0.01$ & $0.11 \pm 0.03$ & $0.17 \pm 0.02$ & $0.08 \pm 0.02$ & $0.14 \pm 0.02$ & $0.07 \pm 0.01$ & $0.27 \pm 0.11$ & $0.18 \pm 0.06$ \\
\hline \multicolumn{9}{|l|}{ ALMERIA YELLOW } \\
\hline Tiempo de secado (Time of drying) (h) & 120 & 840 & 672 & \$ & 168 & 192 & $648^{\circ}$ & $912^{*}$ \\
\hline Incremento de peso (Weight increase) (\%) & $2.58 \pm 0.14$ & $5.09 \pm 0.03$ & $5.47 \pm 0.48$ & $\S$ & $2.10 \pm 0.18$ & $0.85 \pm 0.39$ & $5.94 \pm 1.66$ & $6.82 \pm 0.75$ \\
\hline Descenso de porosidad (Porosity decrease) $(\Delta \%)$ & $1.06 \pm 0.30$ & $3.52 \pm 0.35$ & $6.93 \pm 2.64$ & $\S$ & $0.05 \pm 0.08$ & $1.30 \pm 0.89$ & $6.05 \pm 0.24$ & $5.90 \pm 0.94$ \\
\hline Incremento de densidad (Density increase) $\left(\mathrm{g} \mathrm{cm}^{-3}\right)$ & $0.02 \pm 0.00$ & $0.12 \pm 0.01$ & $0.18 \pm 0.07$ & $\S$ & $0.02 \pm 0.01$ & $0.03 \pm 0.03$ & $0.17 \pm 0.03$ & $0.19 \pm 0.02$ \\
\hline \multicolumn{9}{|l|}{ PUERTO DE SANTA MARIA } \\
\hline Tiempo de secado (Time of drying) (h) & 120 & 840 & 672 & 312 & 264 & 192 & $648^{\circ}$ & $912^{*}$ \\
\hline Incremento de peso (Weight increase) (\%) & $1.60 \pm 0.08$ & $10.15 \pm 0.09$ & $10.80=0.52$ & $1.53 \pm 0.05$ & $3.26 \pm 0.13$ & $1.29 \pm 0.11$ & $10.96 \pm 0.33$ & $9.44 \pm 0.13$ \\
\hline Descenso de porosidad (Porosity decrease) ( $\Delta \%$ ) & $2.17 \pm 0.40$ & $4.01 \pm 0.29$ & $4.31 \pm 0.40$ & $2.04 \pm 0.04$ & $2.70 \pm 0.18$ & $2.56 \pm 0.80$ & $6.03 \pm 0.46$ & $5.89 \pm 0.77$ \\
\hline Incremento de densidad (Density increase) $\left(\mathrm{g} \mathrm{cm}^{-3}\right)$ & $0.08 \pm 0.02$ & $0.08 \pm 0.04$ & $0.05 \pm 0.01$ & $0.02 \pm 0.01$ & $0.06 \pm 0.01$ & $0.05 \pm 0.01$ & $0.10 \pm 0.03$ & $0.09 \pm 0.01$ \\
\hline
\end{tabular}

$\S$ Datos no disponibles (Data no available)

\# Tiempo de secado para el segundo producto (Drying time for the second product applied) 
TABLA III Cont. (TABLE I/I Cont.)

Diversas características de las piedras tratadas

(Diverse characteristcs of the treated stones)

\begin{tabular}{|c|c|c|c|c|c|c|c|c|}
\hline & \multicolumn{8}{|c|}{ 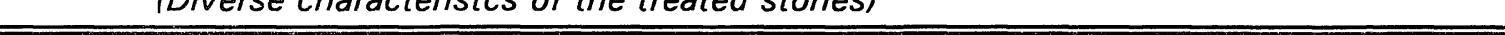 } \\
\hline & \multicolumn{8}{|c|}{ PRODUCTO APLICADO (PRODUCT APPLIED) } \\
\hline & PA & $\mathrm{OH}$ & TV & TG & TGI & ARD & $O B$ & $\mathrm{TT}$ \\
\hline \multicolumn{9}{|l|}{ BAEZA } \\
\hline Tiempo de secado (Time of drying) (h) & 120 & 840 & 672 & 312 & 336 & 192 & $648^{\circ}$ & $912^{\circ}$ \\
\hline Incremento de peso (Weight increase) (\%) & $1.19=0.11$ & $6.86 \pm 0.24$ & $7.45 \pm 0.72$ & $1.64 \pm 1.11$ & $2.44 \pm 0.24$ & $1.87 \pm 0.11$ & $7.761 \pm 0.56$ & $6.782 \pm 0.27$ \\
\hline Descenso de porosidad (Porosity decrease) ( $\Delta \%)$ & $1.25 \pm 0.20$ & $2.44 \pm 0.11$ & $2.92 \pm 0.36$ & $2.16 \pm 0.62$ & $1.43 \pm 1.26$ & $1.69 \pm 0.46$ & $4.07 \pm 0.74$ & $3.92 \pm 0.05$ \\
\hline Incremento de densidad (Density increase) $\left(\mathrm{g} \mathrm{cm}^{-3}\right)$ & $0.03 \pm 0.00$ & $0.03 \pm 0.00$ & $0.03=0.01$ & $0.02 \pm 0.01$ & $0.04 \pm 0.01$ & $0.03 \pm 0.01$ & $0.05 \pm 0.00$ & $0.06 \pm 0.00$ \\
\hline \multicolumn{9}{|l|}{ MARTELLLLA } \\
\hline Tiempo de secado (Time of drying) (h) & 120 & 840 & 672 & 312 & 168 & 192 & $648^{\circ}$ & $912^{*}$ \\
\hline Incremento de peso (Weight increase) (\%) & $0.96 \pm 0.06$ & $5.30 \pm 0.86$ & $5.44 \pm 2.05$ & $0.78 \pm 0.02$ & $2.11 \pm 0.25$ & $1.27 \pm 0.28$ & $4.70 \pm 0.77$ & $5.99 \pm 0.83$ \\
\hline Descenso de porosidad (Porosity decrease) ( $\Delta \%$ ) & $0.32 \pm 0.35$ & $2.60 \pm 0.98$ & $2.46 \pm 0.36$ & $1.20 \pm 0.17$ & $1.77 \pm 0.27$ & $0.52 \pm 0.50$ & $3.84 \pm 1.89$ & $4.86 \pm 0.39$ \\
\hline Incremento de densidad (Density increase) $\left(\mathrm{g} \mathrm{cm}^{-3}\right)$ & $0.03 \pm 0.01$ & $0.03 \pm 0.01$ & $0.03 \pm 0.01$ & $0.03 \pm 0.02$ & $0.04 \pm 0.01$ & $0.01 \pm 0.01$ & $0.09 \pm 0.03$ & $0.11 \pm 0.01$ \\
\hline \multicolumn{9}{|l|}{ PADUL } \\
\hline Tiempo de secado (Time of drying) (h) & 120 & 840 & 672 & 312 & 264 & 192 & $648^{4}$ & $912^{*}$ \\
\hline Incremento de peso (Weight increase) (\%) & $1.09 \pm 0.09$ & $5.97 \pm 0.09$ & $7.31 \pm 1.34$ & $0.67 \pm 0.26$ & $2.02 \pm 0.22$ & $1.96 \pm 0.23$ & $7.49 \pm 1.88$ & $7.05 \pm 0.96$ \\
\hline Descenso de porosidad (Porosity decrease) $(\Delta \%)$ & $0.63 \pm 0.02$ & $3.24 \pm 0.49$ & $3.05 \pm 0.32$ & $1.55 \pm 0.22$ & $2.22 \pm 0.98$ & $1.62 \pm 0.19$ & $5.64 \pm 0.99$ & $5.15 \pm 1.88$ \\
\hline Incremento de densidad (Density increase) $\left(\mathrm{g} \mathrm{cm}^{-3}\right)$ & $0.01 \pm 0.00$ & $0.05 \pm 0.01$ & $0.04 \pm 0.00$ & $0.04 \pm 0.02$ & $0.06 \pm 0.01$ & $0.02 \pm 0.01$ & $0.12 \pm 0.05$ & $0.13 \pm 0.04$ \\
\hline
\end{tabular}

\$ Datos no disponibles (Data no available)

\# Tiempo de secado para el segundo producto (Drying time for the second product applied) 
TABLA IV (TABLE IV)

Cambios de color (oscurecimiento) de las piedras tratadas (Color changes (darkening) of the treated stones)

\begin{tabular}{|c|c|c|c|c|c|c|c|c|}
\hline \multirow[b]{2}{*}{ STONE } & \multicolumn{8}{|c|}{ PRODUCTO APLICADO (PRODUCT APPLIED) } \\
\hline & PA & $\mathrm{OH}$ & TV & TG & TGI & ARD & OB & TT \\
\hline Almería white & 0 & 0 & 0 & 0 & $0 /-$ & + & 0 & 0 \\
\hline Almería yellow & 0 & 0 & 0 & $\S$ & 0 & - & + & + \\
\hline $\begin{array}{l}\text { Puerto de Sta. } \\
\text { María }\end{array}$ & ++ & 0 & 0 & + & + & ++ & + & - \\
\hline Baeza & ++ & 0 & + & ++ & ++ & ++ & ++ & ++ \\
\hline Martelilla & 0 & $0 /-$ & 0 & 0 & - & ++ & 0 & $0 /-$ \\
\hline Padul & + & 0 & - & 0 & 0 & ++ & + & - \\
\hline
\end{tabular}

O No apreciable; - ligero; + considerable; + + muy considerable

10 Not considerable; - slight; + considerable; ++ very considerablel

$\S$ Datos no disponibles (§ Data not available)

de dos productos sobre la piedra. Asimismo, la aplicación de productos que sólo tienen carácter consolidante provoca, en general, un mayor incremento de peso.

En todos los casos se produce un descenso más o menos acusado en el valor de porosidad, correspondiendo los menores descensos a los productos hidrófugos. Los incrementos de densidad son de escasa importancia.

Se han determinado diversos parámetros relacionados con el agua, de acuerdo con las consideraciones del RILEM, cuyos valores aparecen reflejados en la Tabla V.

Como cabía esperar, los mejores valores en lo que respecta a hidrofobicidad corresponden a los productos hidrófugos; no obstante, se puede destacar el buen comportamiento del Strengthener $\mathrm{OH}$ y Tegovakon $\mathrm{V}$, ambos con carácter consolidante solamente, sobre los materiales del Puerto de Santa María.

Paraloid B72 no tiene un buen comportamiento para ninguno de los materiales chequeados, y es posible que sean necesarias mayores concentraciones del producto en solución para el tratamiento de materiales tan porosos como los ensayados. Como se puede ver para el caso de la aplicación de Tegosivin HL100, existen diferencias más que notables para los materiales tratados con una concentración de producto 5 o $10 \%$, con un mejor comportamiento para la concentración más alta. two products were applied. Likewise, the application of the only-consolidant products provokes in general a larger weight increase.

In all the cases a porosity decrease more or less marked was produced and the lower decreases correspond to the water repellent products. The density increases were of small importance.

Diverse properties relationed with the water were measured according to RILEM proposal. Table V shows the main results obtained from the tested materials.

As might have been expected, the best water repellency for the different tests corresponds to those products with hydrophobic characteristics. However, it is remarkable the good behaviour of Strengthener $\mathrm{OH}$ and Tegovakon $V$, with consolidant character only, on Puerto de Santa Maria stone.

Paraloid do not has a good behaviour for the stony materials tested, and it's possible that a higher product concentration must be employed for very porous materials like these; comparing with Tegosivin HL100, as can see on the table, there are very important differences between the materials treated with 5 or $10 \%$ product concentration, with a best behaviour for the higher concentration. 
TABLA V (TABLE V)

Parámetros de absorción de agua para las piedras tratadas (Water-absorption parameters for the treated stones)

\begin{tabular}{|c|c|c|c|c|c|c|c|c|c|}
\hline & \multirow{3}{*}{ UNTREATED } & \multirow{2}{*}{\multicolumn{8}{|c|}{ PRODUCTO APLICADO (PRODUCT APPLIED) }} \\
\hline & & & & & & & & & \\
\hline & & PA & $\mathrm{OH}$ & TV & TG & TGI & ARD & $O B$ & TT \\
\hline \multicolumn{10}{|l|}{ ALMERIA WHITE } \\
\hline $\begin{array}{l}\text { Capilaridad (Capillarity): } \\
\text { Maximum water absorbed }\left(\mathrm{g} \mathrm{cm}^{-2}\right)\end{array}$ & $1.72 \pm 0.07$ & $1.48 \pm 0.10$ & $0.79 \pm 0.33$ & $1.15 \pm 0.62$ & $0.15 \pm 0.05$ & $0.05 \pm 0.03$ & $1.47 \pm 0.07$ & $0.07 \pm 0.022^{\circ}$ & $0.10 \pm 0.03^{*}$ \\
\hline $\begin{array}{l}\text { Inmersión (Immersion): } \\
\text { Maximum water absorbed }\left(\mathrm{mg} \mathrm{cm}^{-3}\right)\end{array}$ & $327.8 \pm 13.5$ & $305.6 \pm 22.3$ & $219.5 \pm 51.2$ & $212.0 \pm 70.2$ & $181.6=3.5^{\circ}$ & $128.9 \pm 49.6^{\circ}$ & $328.0 \pm 21.1$ & $207.1 \pm 42.4^{\circ}$ & $272.1 \pm 6.2^{\circ}$ \\
\hline $\begin{array}{l}\text { Absorción de gota (Drop } \\
\text { absorption): } \\
\text { Time of absorption (min.) }\end{array}$ & $<1$ sec. & $26.3 \pm 3.7$ & $6.4 \pm 6.3$ & $1.0 \pm 0.8$ & $*$ & * & * & $*$ & $*$ \\
\hline \multicolumn{10}{|l|}{ ALMERIA YELLOW } \\
\hline $\begin{array}{l}\text { Capilaridad (Capillarity): } \\
\text { Maximum water absorbed }\left(\mathrm{g} \mathrm{cm}^{-2}\right)\end{array}$ & $1.11 \pm 0.51$ & $1.15 \pm 0.13$ & $.85 \pm 0.32$ & $0.96 \pm 0.08$ & s & $0.05 \pm 0.01$ & $0.13 \pm 0.04^{\circ}$ & $0.05 \pm 0.01^{\circ}$ & $0.06 \pm 0.01^{\circ}$ \\
\hline $\begin{array}{l}\text { Inmersión (Immersion): } \\
\text { Maximum water absorbed }\left(\mathrm{mg} \mathrm{cm}^{-3}\right)\end{array}$ & $258.7 \pm 39.9$ & $220.1 \pm 22.4$ & $201.3 \pm 13.7$ & $195.1 \pm 0.84$ & \$ & $100.1 \pm 3.2^{*}$ & $221.4 \pm 25.4$ & $112.1 \pm 3.5^{\circ}$ & $157.7 \pm 24.1^{*}$ \\
\hline $\begin{array}{l}\text { Absorción de gota (Drop } \\
\text { absorption): } \\
\text { Time of absorption (min.) }\end{array}$ & $<1$ sec. & $4.99 \pm 3.3$ & $48.9 \pm 31.8$ & $0.6 \pm 0.4$ & $\$$ & * & $*$ & * & $*$ \\
\hline \multicolumn{10}{|l|}{ PUERTO DE SANTA MARIA } \\
\hline $\begin{array}{l}\text { Capilaridad (Capillarity): } \\
\text { Maximum water absorbed }\left(\mathrm{g} \mathrm{cm}^{-2}\right)\end{array}$ & $1.42 \pm 0.11$ & $1.11 \pm 0.15^{\circ}$ & $0.03 \pm 0.01$ & $0.06 \pm 0.02$ & $1.07 \pm 0.016$ & $0.03 \pm 0.01$ & $0.23 \pm 0.21^{\circ}$ & $0.08 \pm 0.04^{\circ}$ & $0.07 \pm 0.02^{\circ}$ \\
\hline $\begin{array}{l}\text { Inmersión (Immersion): } \\
\text { Maximum water absorbed }\left(\mathrm{mg} \mathrm{cm}^{-3}\right)\end{array}$ & $299.9 \pm 13.5$ & $276.5 \pm 18.1$ & $259.9 \pm 23.7^{\circ}$ & $217.3 \pm 26.8$ & $264.9 \pm 16.1$ & $177.8 \pm 24.3^{\circ}$ & $242.2 \pm 26.3$ & $133.8 \pm 16.4^{\circ}$ & $259.7 \pm 20.5^{\circ}$ \\
\hline $\begin{array}{l}\text { Absorción de gota (Drop } \\
\text { absorption): } \\
\text { Time of absorption (min.) }\end{array}$ & $<1 \mathrm{sec}$ & ${ }^{*}$ & $0.4 \pm 0.2$ & $61.5 \pm 3.0$ & * & $*$ & $*$ & $*$ & $*$ \\
\hline
\end{tabular}

$\S$ Datos no disponibles (Data no available)

* No absorbida (Not absorbed)

\# Contenido a los 30 días (Content at 30 days) 


\section{TABLA V Cont. (TABLE V Cont.)}

Parámetros de absorción de agua para las piedras tratadas (Water-absorption parameters for the treated stones)

\begin{tabular}{|c|c|c|c|c|c|c|c|c|c|}
\hline & \multirow[b]{2}{*}{ UNTREATED } & \multicolumn{8}{|c|}{ PRODUCTO APLICADO (PRODUCT APPLIED) } \\
\hline & & PA & $\mathrm{OH}$ & TV & TG & TGI & ARD & OB & $\mathrm{TT}$ \\
\hline \multicolumn{10}{|l|}{ BAEZA } \\
\hline $\begin{array}{l}\text { Capilaridad (Capillarity): } \\
\text { Maximum water absorbed }\left(\mathrm{g} \mathrm{cm}^{-2}\right)\end{array}$ & $1.04 \pm 0.01$ & $0.90 \pm 0.06$ & $0.32 \pm 0.10$ & $0.77 \pm 0.01$ & $0.07 \pm 0.01$ & $0.03 \pm 0.01$ & $0.91 \pm 0.04$ & $0.06 \pm 0.01^{\circ}$ & $0.04 \pm 0.01^{\prime}$ \\
\hline $\begin{array}{l}\text { Inmersión (Immersion): } \\
\text { Maximum water absorbed }\left(\mathrm{mg} \mathrm{cm}^{-3}\right)\end{array}$ & $203.0 \pm 3.2$ & $192.9 \pm 17.6$ & $210.7 \pm 15.2$ & $107.4 \pm 87.8$ & $112.2=4.2^{\circ}$ & $86.6 \pm 0.1$ & $198.9 \pm 12.8$ & $109.1 \pm 8.8^{*}$ & $131.1 \pm 7.0^{\circ}$ \\
\hline $\begin{array}{l}\text { Absorción de gota (Drop } \\
\text { absorption): } \\
\text { Time of absorption (min.) } \\
\end{array}$ & $<1 \mathrm{sec}$ & * & $1.16 \pm 0.72$ & $26.18 \pm 11.43$ & * & * & * & $*$ & $46.59 \pm 9.31$ \\
\hline \multicolumn{4}{|l|}{ MARTELILLAA } & & & & & & \\
\hline $\begin{array}{l}\text { Capilaridad (Capillarity): } \\
\text { Maximum water absorbed }\left(\mathrm{g} \mathrm{cm}^{-2}\right)\end{array}$ & $1.10 \pm 0.14$ & $0.90 \pm 0.23$ & $0.20 \pm 0.04$ & $0.76=0.47$ & $0.06 \pm 0.01$ & $0.02 \pm 0.00$ & $0.37 \pm 0.20^{\prime \prime}$ & $0.05 \pm 0.01^{\prime}$ & $0.14 \pm 0.03^{\circ}$ \\
\hline $\begin{array}{l}\text { Inmersión (Immersion): } \\
\text { Maximum water absorbed }\left(\mathrm{mg} \mathrm{cm}^{-3}\right)\end{array}$ & $211.0 \pm 8.6$ & $165.9 \pm 37.3$ & $204.6 \pm 25.4$ & $179.3 \pm 28.4$ & $72.3 \pm 1.4$ & $55.0 \pm 5.5^{\circ}$ & $213.7 \pm 14.8$ & $55.17 \pm 3.7^{\circ}$ & $193.63 \pm 49.1^{\circ}$ \\
\hline $\begin{array}{l}\text { Absorción de gota (Drop } \\
\text { absorption): } \\
\text { Time of absorption (min.) }\end{array}$ & $<1 \mathrm{sec}$ & $79.5=53.5$ & $34.3 \pm 15.1$ & $15.2 \pm 7.7$ & * & * & * & * & $*$ \\
\hline \multicolumn{10}{|l|}{ PADUL } \\
\hline $\begin{array}{l}\text { Capilaridad (Capillarity): } \\
\text { Maximum water absorbed }\left(\mathrm{g} \mathrm{cm}^{-2}\right)\end{array}$ & $1.10 \pm 0.13$ & $0.87 \pm 0.13^{\circ}$ & $0.20 \pm 0.07$ & $0.38 \pm 0.04$ & $0.30 \pm 0.14$ & $0.04 \pm 0.01$ & $0.91 \pm 0.10^{\circ}$ & $0.02 \pm 0.00^{\circ}$ & $0.08 \pm 0.01^{\prime}$ \\
\hline $\begin{array}{l}\text { Inmersión (/mmersion): } \\
\text { Maximum water absorbed }\left(\mathrm{mg} \mathrm{cm}^{-3}\right)\end{array}$ & $207.7 \pm 24.5$ & $191.6=19.5$ & $199.3 \pm 12.1^{\circ}$ & $147.3 \pm 18.9$ & $177.0 \pm 18.1$ & $113.7 \pm 6.8^{\circ}$ & $196.5 \pm 8.5$ & $141.5=32.3^{\circ}$ & $199.4 \pm 27.6$ " \\
\hline $\begin{array}{l}\text { Absorción de gota (Drop } \\
\text { absorption): } \\
\text { Time of absorption (min.) }\end{array}$ & $<1 \mathrm{sec}$ & $10.5 \pm 4.9$ & $7.5 \pm 2.9$ & $5.3 \pm 3.0$ & $*$ & $*$ & $*$ & $*$ & * \\
\hline
\end{tabular}

§ Datos no disponibles (Data no available)

* No absorbida (Not absorbed)

\# Contenido a los 30 días (Content at 30 days) 
El producto acrilsilicónico (ARD), presenta un comportamiento intermedio entre Paraloid y los productos hidrófugos de tipo silicónico. Por último, sólo basta decir que el mejor comportamiento se produce con los materiales son tratados con Tegosivin HL100 (10\%), y cuando se aplicaron dos productos de forma correlativa (ambos silicónicos).

\section{CONCLUSIONES}

Los resultados obtenidos revelan que los tratamientos que proporcionan un mejor comportamiento de tipo hidrofóbico son los de tipo silicónico: Tegosivin HL100 (10\%) y la aplicación correlativa de Strengthener OH/BS 28 y Tegovakon/Tegosivin (10\%). No obstante, para una aplicación real en un monumento, deben tenerse en cuenta otros factores de alteración que puedan estar presentes, tales como procesos de ataque químico, cristalización de sales, cambios termohigrométricos, etc. En este sentido, actualmente, los pares producto/piedra están siendo sometidos a procesos de alteración acelerada, a fin de obtener la mejor combinación para cada problema de conservación, constituyendo los resultados obtenidos el núcleo de futuras publicaciones.
The acrylsiliconic product (ARD) has an halfway behaviour between Paraloid and the siliconic-water repellent products, and the best behaviour corresponds to Tegosivin (10\%) and when the materials were treated with two products (both siliconic).

\section{CONCLUSIONS}

The results obtained reveal that the best treatments to apply with hydrophobic aim to the materials tested are the siliconic ones: Tegosivin HL100 (10\%) and the correlative application of Strengthener OH/BS 28 and Tegovakon/Tegosivin (10\%). However, it must be considered other alteration factors which actuate on the monuments; so, processes of chemical attack, salt crystallization, thermohygrometric changes, etc must be considered. In this way, nowadays all the pairs Product/stone are submitting to accelerated alteration processes with the aim to obtain the best combination for each conservation problems, even in the same building and they will constitute future publications.

\section{AGRADECIMIENTOS}

Este trabajo ha sido realizado con una subvención de la DICYT (PAT90-0929).

\section{ACKNOWLEDGEMENT}

This work has been realized with a DICYT subvention (PAT90-0929).

\section{BIBLIOGRAFÍA}

(1) AMOROSO, G.G. AND FASSINA, V.: "Stone Decay and Conservation", Ed. Elsevier, Amsterdam, 1983.

(2) LEWIN, S.Z.; CHAROLA, A.E.: (1979) "Scanning electron microscopy in of 'diseased' stone", Scanning Electron Microscopy, Vol. 1, 1979, 695-703.

(3) MARTÍN, A.: "Ensayos y experiencias de alteración en la conservación de obras de piedra de interés histórico artístico", Ed. C.E.U.R.A., Madrid, 1990.

(4) MARTÍN, L.; BELLO, M.A.; MARTÍN, A.: “Alteration mechanisms of the stones used in the Cathedral of Granada (Spain)", Materiales de Construcción, Vol 43, No. 229, 1993, 25-33.

(5) RILEM Commission 25-PEM "Protection et érosion des monuments", Ed. Bordas-Dunod, Paris, 1978.

(6) BERZOSA, J.; MARTÍN, A.: “Análisis rápido de materiales calizos y silíceos.I.Determinación de sílice”, Ensayos e Investigación, 1(3), 1966, 12-18.

(7) BERZOSA, J.; MARTÍN, A.: “Análisis rápido de materiales calizos y silíceos.III. Determinación de calcio, magnesio, sodio y potasio", Ensayos e Investigación, 1(5), 1966, 16-29.

(8) BERZOSA, J.; MARTÍN, A.: “Análisis rápido de materiales calizos y silíceos.II. Determinación de aluminio, hierro y titanio", Ensayos e Investigación, 1(4), 1966, 12-24.

(9) BELLO, M.A.; MARTÍN, A.: "Corrosion processes (chemical deterioration) on the stones used in the Cathedral of Seville (Spain)", Materiales de Construcción, Vol 40, no. 217, 1990, 17-37. 
(10) MARTÍN, L.; BELLO, M.A.; MARTÍN, A.: "The efflorescences of the Cathedral of Almeria (Spain)", in Proceedings 7th Intern. Cong. on Deterioration and Conservation of Stone, Lisboa (Portugal) 1992, 869-873.

(11) CHAROLA, A.E.; WHEELER, G.E.; FREUND, G.: "The influence of relative humidity in the polymerization of methyltrimetoxilane", in Adhesives and Consolidants, Paris, 1984, 177-181.

(12) LEWIN, S.Z.; WHEELER, G.E.: "Alkoxysilane chemistry and stone conservation", in 5th Int. Cong. on Deterioration and Conservation of Stone, Lausanne, 1985, 831-844.

(13) PAULY, J.P.: “Qualité d'un traitment et teneur en eau", in 5th Int. Cong. on Deterioration and Conservation of Stone, Lausanne, 1985, 817-829.

\section{publicaciones del IETCC/CSIC}

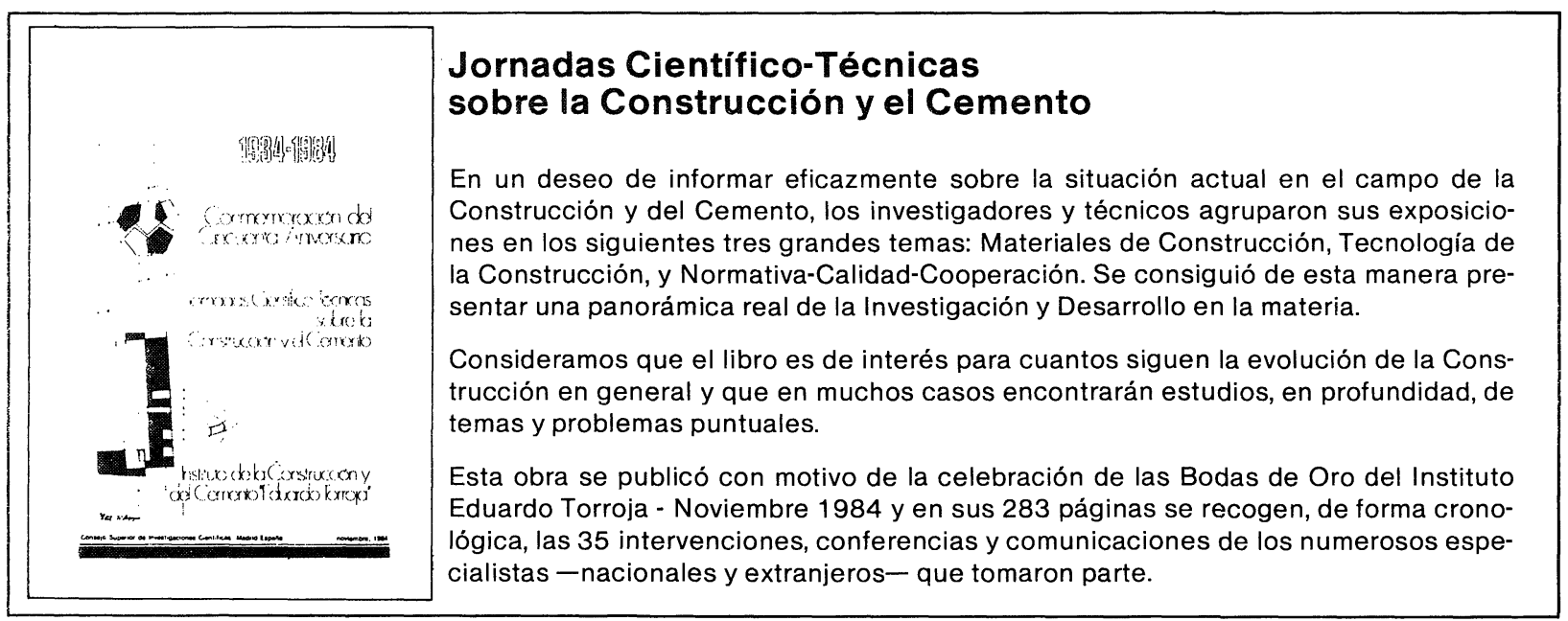

\section{ACUEDUCTOS ROMANOS EN ESPAÑA Carlos Fernández Casado} Prof. Dr. Ing. de Caminos, Canales y Puertos

Esta publicación se compone de una serie de articulos, publicados en la Revista "Informes de la Construcción", en los cuales se hace un análisis de los acueductos romanos que existen en Espafia y el balance de las condiciones de conservación en que se encuentra cada uno de ellos, incluyendo referencias históricas y literarias. Se ha ilustrado con la reproducción de la valiosa documentación gráfica que posee el prestigioso autor.

Un volumen encuadernado en couché, a dos colores, de $21 \times 27$ centimetros, compuesto de 238 páginas, numerosos grabados, dibujos, fotos en blanco y negro y figuras de linea.

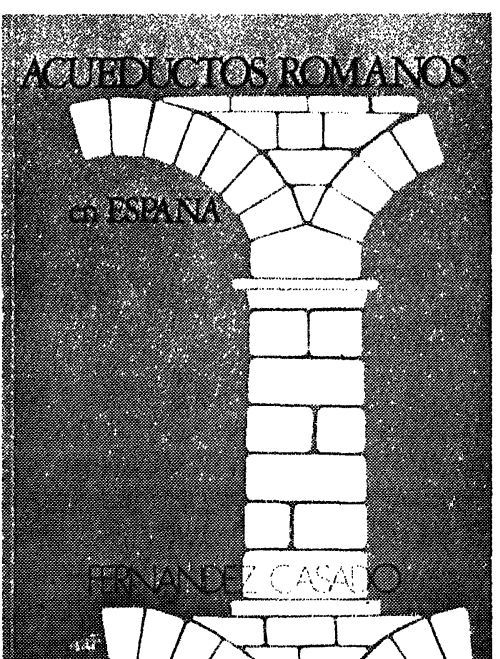

